\title{
APROXIMACIÓN AMBIENTAL A LAS LAGUNAS ENDORREICAS PROTEGIDAS DE LA PROVINCIA DE SEVILLA (ESPAÑA).
}

\author{
F.J. ORTIZ BOTELLA* \\ Jesús MOLINA VÁZQUEZ *
}

\section{NTRODUCCIÓN}

Pese a los esfuerzos de conservación que en los últimos decenios se vienen desarrollando para la protección de las zonas húmedas, el conocimiento sobre muchas de ellas es escaso e insuficiente a la hora de plantear modelos de gestión adecuados para su preservación, debido en gran medida a la consideración tradicional como áreas improductivas y dañinas.

En el caso de las lagunas y complejos endorreicos de la provincia de Sevilla la información sobre las mismas es parcial y dispersa.

MADOZ, a mediados del siglo pasado, aporta datos útiles, principalmente sobre las medidas morfométricas de algunos de los espacios y nos aproxima a una concepción no muy lejana en el tiempo. Refiriéndose a la laguna de Calderón, en Osuna, comenta: “...es la notable que hay en el término, se extiende sobre $460 \mathrm{fa}$ negas de tierra y dista 2 leguas: en el verano ocasiona grandes males en los habitantes de las casas de campo, que cultivan el terreno de sus inmediaciones; mas éstas cesarán si un rico hacendado de esta villa lleva á cabo el pensamiento de desaguarla, con lo que haría un gran beneficio a la humanidad y al país" (sic.) (Pascual Madoz. Diccionario Geográfico Histórico Estadístico de España y sus posesiones de Ultramar. Sevilla. Edición facsímil. Ambito Ediciones. pp: 124).

MACHADO, en 1854, estudia la avifauna de la provincia de Sevilla y, aunque se centra fundamentales en las marismas de Doñana y el río Guadalquivir, algunas de sus referencias son de interés. Ya a principios de este siglo REYES PROSPER, en su obra sobre las estepas ibéricas, visita diversas lagunas de la provincia, dejando constancia de su riqueza botánica.

\footnotetext{
* Bí́logos.
} 
No será hasta mediados del presente siglo, en 1940, cuando DANTIN realice una aproximación geográfica al fenómeno del endorreismo bético, estudiando específicamente más de 140 lagunas andaluzas. Entre ellas se describen cerca de 60 lagunas de la provincia. Posteriormente PARDO recoge la información existente hasta la fecha en su Catálogo de los lagos de España.

A principios de los años 60, comienzan los censos nacionales de acuáticas coordinados por la SEO, y el conocimiento sobre las avifauna de las lagunas y su importancia empieza a ser valorada. En particular los censos sobre aves invernantes son abundantes y continuados, bien para las lagunas estudiadas o en el ámbito de los humedales andaluces (BERNIS y VALVERDE, 1968; BERNIS, 1972; HAFNER y WALMSLEY, 1974; ARAUJO, 1978; SÁNCHEZ MORENO, 1979; GARCÍA et al 1980; ENA y PURROY, 1982; SEO, 1984; SEO, 1985; SEO, 1987a, 1987b; SEO, 1990).

Sin embargo, es en los últimos años cuando la preocupación por la conservación de las zonas húmedas se ha traducido en investigaciones que contemplan el fenómeno de las lagunas interiores desde otras perspectivas, que han contribuido a obtener una visión más global y acertada sobre estos ecosistemas. Así se han abarcado aspectos históricos y de conservación (FAJARDO y MAZUELOS, 1983, 1993; ORTIZ y MOLINA, 1995) geográficos y geomorfológicos (DIAZ DEL OLMO et al., 1990; RECIO ESPEJO, 1989), ecológicos y bióticos (FUREST y TOJA, 1984, 1987; AMAT, 1984a; AMAT, 1984b; MONTES, AMAT y RAMIRES DIAZ, 1982a, 1982b) o de riesgos ecológicos potenciales (CONTRERA DE VERA et al., 1989).

La última aportación a reseñar es la Guía de los lagos y humedales de España (CASADO y MONTES, 1995) donde, pese a la extensión del ámbito estudiado, se pueden encontrar diversos datos sobre las lagunas de la provincia.

\section{CARACTERÍSTICAS DEL MEDIO}

En la actualidad son cuatro los espacios endorreícos protegidos en la provincia de Sevilla, bajo la categoría de Reserva Natural (según la Ley 2/89 de Espacios Naturales Protegidos de Andalucía): Complejo Endorreíco de Utrera, Complejo Endorreíco de Lebrija-Las Cabezas, Complejo Endorreíco de la Lantejuela y, por último, laguna de El Gosque.

Situados en la mitad suroriental de la provincia (FIGURA 1) son, en general, lagunas de pequeñas dimensiones (TABLA 1), localizadas en áreas de topografia suave, donde las pendientes no suelen superar el $10 \%$.

En detalle pueden considerarse cuatro sectores endorreicos bien definidos concordantes con los distintos espacios protegidos: 
FIGURA 1

LOCALIZACIÓN GEOGRÁFICA DE LAS LAGUNAS PROTEGIDAS DE LA PROVINCIA DE SEVILLA, DAJO LA CATEGORÍA DE RESERVA NATURAL
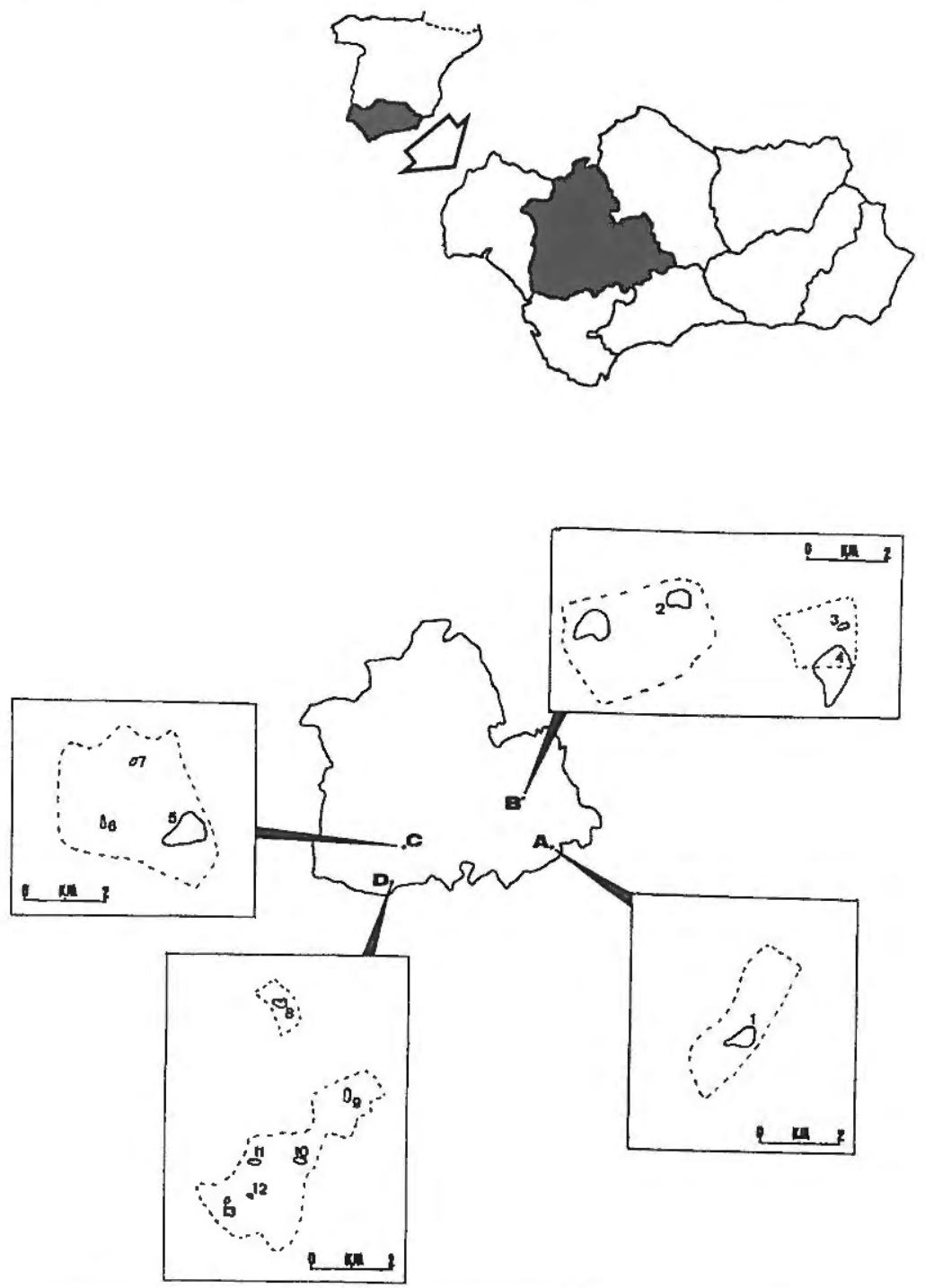

A) Laguna de El Gosque; B) Complejo Endorreico de La Lantejuela; C) Complejo Endorreico de Utrera; D) Complejo Endorreico de Lebrija-Las Cabezas. 1: El Gosque; 2: Ballestera; 3: Calderón Chica; 4: Hoya de Calderón (transformada); 5) Zarracatín; 6: Arjona; 7: Alcaparrosa; 8: Charroao; 9; Taraje; 10: Pilón; 11: Peña; 12: Galiana; 13: Cigarrera.

La línea discontínua representa el límite de la Zona de Protección. Basado en A.M.A. (1990). 
TABLA I

CARACTERÍSTICAS MORFOMÉTRICAS DE LAS LAGUNAS DE LA PROVINCIA DE SEVILLA

\begin{tabular}{|c|c|c|c|c|c|}
\hline LAGUNA & $\begin{array}{l}\mathrm{LM} \\
\mathrm{mts}\end{array}$ & $\begin{array}{l}\mathrm{AM} \\
\mathrm{mts}\end{array}$ & $\begin{array}{c}\text { S } \\
\text { has }\end{array}$ & $\begin{array}{c}\mathrm{P} \\
\mathrm{mts}\end{array}$ & $\begin{array}{l}\mathrm{SC} \\
\text { has }\end{array}$ \\
\hline Taraje ................................. & 430 & 240 & 8,4 & 2,0 & 202 \\
\hline 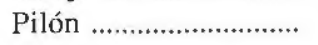 & 370 & 210 & 10,9 & 0,6 & 77 \\
\hline 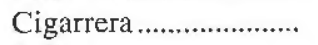 & 215 & 190 & 6,5 & 1,2 & 102 \\
\hline Peña .............................. & 250 & 195 & 4,7 & 1,0 & 115 \\
\hline Galiana ................................ & 160 & 140 & 2,5 & 0,4 & 33 \\
\hline 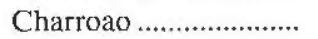 & 545 & 75 & 5,8 & 0,3 & 76 \\
\hline 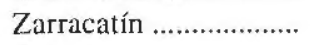 & 1.150 & 860 & 72,2 & 0,2 & 336 \\
\hline Arjona & 250 & 115 & 4,4 & 1,3 & 203 \\
\hline Alcaparrosa .................... & 310 & 150 & 5,6 & 1,0 & 171 \\
\hline Calderón Chica ........... & 310 & 24.5 & 6,9 & 0,2 & 130 \\
\hline 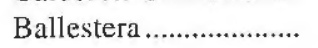 & 635 & 620 & 32,3 & 0,2 & 104 \\
\hline 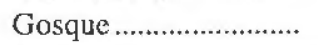 & 815 & 445 & 45,2 & 1,0 & - \\
\hline
\end{tabular}

LM: Longitud máxima; AM: Anchura máxima; S: Superficie de la laguna; P: Profundidad; (*) SC: Superficie de la cuenca.

Fuente: AMAT (1984a) y (*) Elaboración propial

El complejo endorreico de Lebrija-Las Cabezas forma parte de un foco endorreico de mayor escala dimensional que se extiende por la provincia de Cádiz (Complejo endorreico de Espera). Comprende las lagunas de Pilón, Charroao, Peña, Galiana, Cigarrera y Taraje. En su génesis se asocian la presencia de materiales triásicos de base, que forman un substrato impermeable, y un escaso o nulo desarrollo de la red de drenaje. A ello se unen otros rasgos morfogenéticos, tales como fenómenos de disolución y factores estructurales, que favorecieron la formación de pequeñas cubetas semicerradas posteriormente drenadas (COLÓN DÍAZ y DÍAZ DEL OLMO, 1990).

El Complejo endorreico de Utrera comprende tres lagunas, Arjona, Alcaparrosa y Zarracatín, destacando esta última, tanto por sus dimensiones como por su carácter salino. Localizada sobre materiales triásicos, su génesis es explicada por la impermeabilidad del sustrato, lo que favorece el mal drenaje de la zona (IGME, 1988). Aún así deben contemplarse, al igual que en otras áreas similares, la presencia de procesos disolutivos y kársticos.

El complejo endorreico de la Lantejuela formado en la actualidad por las lagunas de Calderón Chica y Ballestera, es una pequeña muestra de lo que hasta mediados de siglo era una extensa zona endorreica, con lagunas tan importantes como Ruíz Sánchez y Calderón. En su origen intervinieron diversos factores geológicos 
de crácter regional, como la evolución de la red hidrográfica del Guadalquivir, con grandes movimientos a través del Cuaternario, y la tectónica regional, con movimientos Pliocuaternarios del Olitostroma. Todo ello condicionó la formación de un amplio episodio de carácter lacustre, donde dominaban lagos en régimen de alternancia, procesos de edafización más o menos marcados y débiles arroyadas. El cambio hacia las condiciones actuales se desarrolla a partir de un proceso de inversión del relieve, acentuado localmente por procesos disolutivos de los materiales margosos (DÍAZ DEL OLMO y RECIO ESPEJO, 1991).

Por último, la laguna del Gosque se ubica en la región geológica Subbética y está asociada al foco endorreíco de la provincia de Málaga, del que la laguna de Fuente de Piedra es su máxima espresión. Estas lagunas constituyen formaciones kársticas originadas por la disolución de los materiales de base (MONTES y MARTINO, 1987).

El régimen de inundación de las lagunas estudiadas depende de las condiciones climáticas, reponsables tanto de los aportes superficiales de agua como de los subsuperficiales y subterráneos procedentes de sus respectivas cuencas hidrográficas. En este punto cabe hacer una pequeña reflexión sobre la función que tradicionalmente se les ha asignado a las aguas subterráneas en las lagunas endorreicas. Así el concepto clásico de endorreismo como zonas donde el agua de lluvia se acumula por ausencia de salida al mar debe ser revisado.

Se ha puesto de manifiesto como la circulación subterránea de agua juega un papel destacado tanto en los ecosistemas terrestres como en los humedales. La permanencia de agua en las lagunas con cuencas superficiales de recepción muy pequeñas puede deberse a flujos más o menos importantes de aguas subterráneas. Aunque en la mayoría de los casos el flujo ascendente produce un encharcamiento pasajero y, a veces, solamente un humedecimiento del sustrato, éste puede tener gran importancia al impedir la infiltración de las aguas de lluvias y de escorrentía que se acumulan, por tanto, en la depresión lagunar (GONZÁLEZ BERNÁLDEZ, 1987).

Por otro lado, la circulación de las aguas por el subsuelo y el tiempo de permanencia en el mismo (flujos locales y regionales), son elementos básicos para entender, en muchos casos, no sólo la cantidad de agua que mantienen las lagunas, sino también su grado de mineralización, ante la presencia de masas de agua cuya composición química no concuerda con las características litológicas de su cuenca (MONTES y MARTINO, 1987).

En este contexto, las lagunas de Taraje y El Gosque merecen especial interés. La primera por ses la única de las estudiadas de carácter permanente, en la que los aportes subterráneos son desconocidos, si bien pueden ser fundamentales en el comportamiento hídrico de la misma. La del Gosque por estar localizada sobre un acuífero de cierta extensión, Acuífero de Martín de la Jara-Los Corrales. 


\section{VALOR FAUNÍSTICO Y BOTÁNICO}

La importancia ecológica de las zonas húmedas interiores como áreas de cría, reproducción y migración para numerosas especies de avifauna es de sobra conocida, siendo en muchas ocasiones el principal factor considerado a la hora de dotar de protección a estos espacios. Este hecho adquiere en este caso especial relevancia por su posición cercana a áreas como las marismas de Doñana, las zonas húmedas de la provincia de Cádiz o la Laguna de Fuente de Piedra.

Debido al carácter estacional de la mayoría de las lagunas, el uso del espacio por las aves acuáticas no es constante y aparece condicionado tanto por el nivel de encharcamiento, como por la disponibilidad de alimento o la presencia de cinturones de vegetación perilagunar bien conservados.

El número de especies en las lagunas sevillanas oscila ampliamente, siendo la de Galiana la que presenta menor diversidad, y las de Taraje y El Gosque las que mayor número registran (AMAT, et al 1985).

Atendiendo a su abundancia, son lás anátidas y los rálidos las especies más frecuentes en los diversos complejos. Sin embargo, no deben olvidarse la presencia de especies de especial interés como la malvasía (Oxyura leucocephala), la focha cornuda (Fulica cristata), o el flamenco (Phoenicopterus ruber), existiendo diversas citas al respecto (BLASCO, LUCENA y RODRÍGUEZ, J., 1979; ARENAS GONZÁLEZ y AYALA MORENO, 1986; CEBALLOS BENITO, 1987; A.M.A. CORDOBA, 1991; FERNÁNDEZ-PALACIOS y RAYA, 1991; PEREIRA SIESO, 1991; RAMÍREZ, RENDON y VARGAS, 1992; TORRES ESQUIVIAS et al. 1994).

Cabe apuntar aquí la inclusión de la laguna del Taraje como Zona de Especial Protección para las Aves (ZEPA) según la directiva adoptada por el Consejo de las Comunidades Europeas de conservación de aves silvestres (Directiva 79/409/CEE).

Pero la trascendencia ecológica de las Iagunas de la provincia no se circunscribe tan sólo en su interés avifaunístico, pese a ser el más conocido, destacando también su relevancia para otros grupos bióticos. Tal es el caso de numerosas especies botánicas que se desarrollan en estos ecosistemas. Bajo este punto de vista las lagunas sevillanas adquieren una dimensión poco conocida.

El caso más significativo es el del macrófito Althenia orientalis, presente en varias de las lagunas estudiadas (ALONSO, COMELLES y MARGALEF, 1980; SILVESTRE y CABEZUDO, 1980; TALAVERA, AMAT y FUREST, 1984) y que es considerada como especie de máximo riesgo de extinción en Andalucía (HERNÁNDEZ BERMEJO y CLEMENTE MUÑOZ, 1994). Este carácter de refugio botánico es ampliable a otras especies que se desarrollan en algunos espacios como Riella helycophylla, Ruppia drepanensis o Zannichellia obtusifolia las cuales, procedentes de las antiguas comunidades esteparias, pueden competir con éxito en los sistemas lagunares (GARCÍA MURILLO, 1991). 
Además las lagunas interiores representan un hábitat adecuado para otros grupos, en particular invertebrados acuáticos, que adaptados a sus peculiares condiciones de inestabilidad se desarrollan en estos ecosistemas, siendo en general insuficientemente conocidos.

\section{SU VALOR CULTURAL}

Una vez comentado el valor biológico de las lagunas de la provincia, merece la pena detenerse, aunque sea de modo somero, en ciertos aspectos culturales, tanto por ser una dimensión poco conocida de las lagunas interiores como por su carácter histórico.

En general las condiciones salinas de gran parte de las lagunas sevillanas ha sido factor determinante tanto para su conservación, como para el desarrollo de ciertos usos económicos y sociales.

La alta concentración de sal ha provocado, en muchos casos, una escasa utilidad de sus cubetas para fines agrícolas, siendo precisamente las menos salinas las que han sufrido más importantes procesos de transformación y desecación (MONTES y MARTINO, 1987).

Así mismo, la extracción de sal a partir de las lagunas interiores ha sido una práctica habitual, recogiéndose las sales que quedaban depositadas en los humedales durante su periodo de desecación. Esta actividad tuvo cierta trascendencia económica en la laguna de El Gosque, donde a mediados del siglo pasado se localizaba una fábrica destinada a la explotación de la sal cuajada, que posteriormente era expendida en los alfolíes de la empresa arrendataria (MADOZ, 1945).

Más corriente, era la obtención de sales de las agnas subterráneas mediante la construcción de pozos, encontrándose ejemplos de estas instalaciones tradicionales en las proximidades de La Lantejuela (CASADO y MONTES, 1995).

También asociado al carácter salino de las lagunas se sitúa la consideración medicinal que tradicionalmente han tenido sus aguas. El uso de El Gosque como zona de baño, donde se podían curar las enfermedades de la piel, era una práctica habitual entre los habitantes de Martín de la Jara hasta muy recientemente.

\section{ESTADO AMBIENTAL DE LAS LAGUNAS}

A fin de obtener una visión de la calidad medioambiental de las lagunas de la provincia de Sevilla se ha establecido una guía de los principales impactos antrópicos a los que se están sometidas. Para ello, entre mediados de 1993 y principios de 1994 se realizaron reconocimientos de campo con el objetivo de detectar los principales problemas que afectan a las mismas. 
La tipificación de impactos se basa en trabajos anteriores realizados en las lagunas y complejos endorreícos de la provincia de Cádiz (DÍAZ DEL OLMO, MOLINA VÁZQUEZ y SANTO ROSA, 1985). Cada impacto fue valorado en una escala de orden creciente negariva (TABLA II). En base a este listado y al reconocimiento de las lagunas se ha confeccionado una matriz de impactos (TABLA III), que facilita una visión global de los diferentes espacios.

\section{TABLA II \\ TIPOLOGÍA DE IMPACTOS ESTABLECIDA PARA LAS LAGUNAS PROTEGIDAS DE LA PROVINCIA DE SEVILLA}

1. Afluencia de visitantes:

+ No hay

1 Visitas sin repercusión

2 Repercusión en las nidificantes

3 Fuertes molestias para el desarrollo de la avifauna, lo que provoca desplazamientos de las comunidades a otros puntos

2 y 3. Actividades agrícolas de secano y regadío

+ No existe

1 Agricultura sin apreciables repercusiones en la laguna

2 Agricultura con repercusiones sin llegar al nivel 3

3 Agricultura con apreciables repercusiones en la laguna (Invasión del vaso lagunar, alteración del régimen hídrico, etc.)

4. Degradación de la vegetación perilagunar

+ Existe cinturón de vegetación bien conservado

1 Cinturón perilagunar degradado en algunas zonas

2 Cinturón altamente degradado con repercusiones sobre la avifauna y la laguna.

3 Cinturón perilagunar inexistente

5. Carga ganadera

+ No hay

1 Existen infraestructuras en desuso o antiguas actuaciones que no han alterado el régimen hídrico normal

2 Existen infraestructuras en uso esporádico o pasadas actuaciones que han alterado eI régimen hídrico natural

3 Existen infraestructuras en uso continuado o actuaciones que siguen alterado el régimen hídrico normal

7. Extracciones de aguas (superficiales o subterráneas) en el ámbito de la laguna

+ No existen

1 Existen sin repercusiones

2 Existen con leves repercusiones

3 Existen alterando sensiblemente el régimen hídrico 
8. Vertidos sólidos

+ No existen

1 Vertidos esporádicos sin repercusiones

2 Vertidos esporádicos o antiguos vertidos que pueden alterar las características de la laguna

3 Vertidos esporádicos o constantes que alteran las características naturales

9. Contaminación y eutrofización

+ No existe

1 Presencia de contaminantes sin efectos

2 Presencia de contaminantes que provocan cambios en la laguna sin llegar al nivel 3

3 Eutrofización total y paulatina de las aguas

10. Erosión en la cuenca

+ No hay

1 Afectan al entomo sin generar procesos de colmatación en el área de encharcamiento

2 Afectan al entorno provocando procesos de colmatación sin llegar al nivel 3

3 Alto proceso de colmatación que provoca desecaciones

11. Infraestructuras (Carreteras, merenderos, tendidos eléctricos, etc)

+ No existen

1 Existen en el entorno sin repercusiones

2 Existen con alto riesgo de afectar las condiciones actuales

3 Existen con repercusiones sobre las comunidades bióticas o las condiciones naturales

Evidentemente esta metodología posee algunos inconvenientes. La diversidad de situaciones implica que en cada laguna las causas de un impacto sean distintas. Por otro lado la ausencia de estudios específicos impide conocer con exactitud la capacidad de respuesta del sistema y de sus distintos componentes ante una actuación. Por último cada impacto considerado puede tener repercusiones, directas o indirectas, sobre otros componentes del ecosistema que no son considerados en el listado confeccionado.

Pese a las insuficiencias del método puede ser un instrumento útil para la conservación de dichos espacios, al permitir evaluar de un modo sencillo los efectos que las medidas de gestión puedan tener, utilidad ya puesta de manifiesto en los humedales gaditanos (MOLINA VÁZQUEZ, 1991).

Así mismo, con objeto de realizar una aproximación gráfica a la matriz obtenida se realizó un análisis de cluster (según Indice de Similaridad de Bray-Curtis) a fin de agrupar las lagunas en función de los diferentes impactos que les afectan. El resultado se representa en la FIGURA II y, como puede observarse, las lagunas aparecen reunidas independientemente de su pertenencia a un mismo foco endorreico. 


\section{TABLA III}

PRINCIPALES IMPACTOS Y RIESGOS A LOS QUE SE ENCUENTRAN SOMETIDAS LAS LAGUNAS ESTUDIADAS

\begin{tabular}{|c|c|c|c|c|c|c|c|c|c|c|c|}
\hline & 1 & 2 & 3 & 4 & 5 & 6 & 7 & 8 & 9 & 10 & 11 \\
\hline GOSQUE ................... & 2 & 2 & 3 & 1 & + & 2 & 3 & 3 & 3 & 2 & 3 \\
\hline ZARRACATIN .... & + & 2 & + & 3 & + & + & 1 & + & 2 & 2 & 2 \\
\hline ARJONA …................ & + & 1 & + & + & + & + & 1 & + & 2 & 1 & 2 \\
\hline ALCAPARROSA. & + & 1 & 3 & 1 & + & 3 & 1 & + & 3 & 1 & 2 \\
\hline BALLESTERA .... & + & 1 & 1 & 2 & + & + & 1 & 2 & 2 & 2 & 1 \\
\hline CALDERON ......... & + & 1 & + & 3 & + & + & + & + & 2 & 1 & 2 \\
\hline TARAJE .................. & + & 1 & + & 1 & + & 1 & 1 & + & 1 & 2 & 1 \\
\hline CIGARRERA ........ & + & 1 & + & + & 1 & + & 1 & + & 1 & 1 & 1 \\
\hline GALIANA ............... & + & 1 & + & 2 & + & + & 1 & + & 1 & 1 & 1 \\
\hline PEÑA & + & 1 & + & 2 & + & 1 & + & + & 1 & 1 & 1 \\
\hline CHARROAO ........ & + & 3 & + & 3 & + & + & + & + & 1 & 2 & 1 \\
\hline PILON & + & 1 & + & 1 & + & + & + & + & 1 & 1 & 3 \\
\hline
\end{tabular}

1: Afluencia de visitantes; 2: Actividad agrícola de secano; 3: Actividad agrícola de regadío; 4: Degradación de la vegetación perilagunar; 5: Carga ganadera; 6: Drenajes artificiales o actuaciones que alteran el régimen hídrico; 7: Extracciones de aguas en el ámbito de la laguna; 8: Vertidos sólidos; 9: Contaminación y eutrofización; 10: Erosión en el cuenca; 11: Infraestructuras.

\section{FIGURA II}

DENDOGRAMA RESULTANTE EN BASE A LA MATRIZ DE IMPACTOS OBTENIDA PARA LAS LAGUNAS ESTUDIADAS SEGÚN ÍNDICE DE SIMILARIDAD DE BRAY-CURTIS

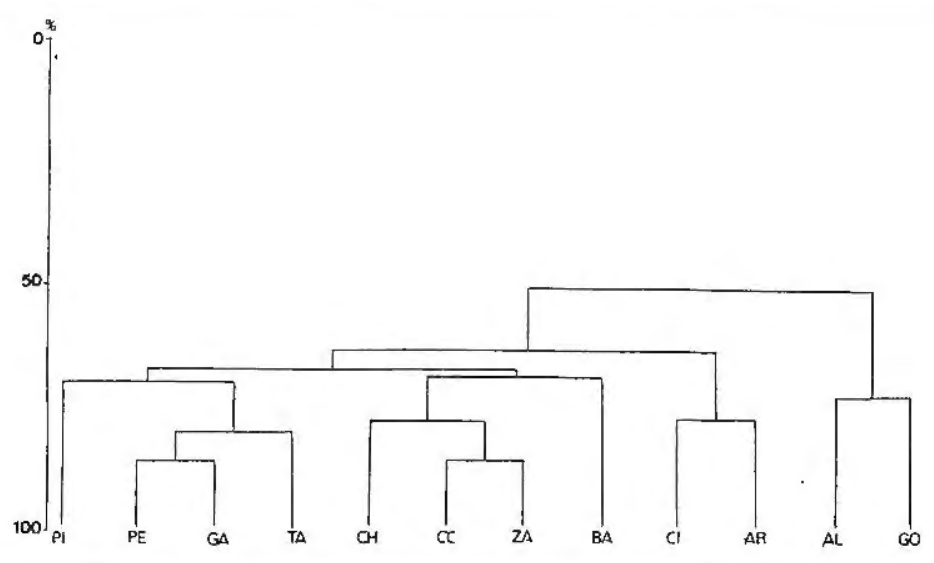

El algoritmo de agrupación utilizado fue el UPGMA (Unweigted pairgroup method using arithmetic average). PI: Pilón; PE: Peña; GA: Galiana; TA: Taraje; $\mathrm{CH}$ : Charroao; CC: Calderón Chica; ZA: Zarracatín; BA: Ballestera; CI: Cigarrera; AR: Arjona; AL: Alcaparrosa; GO: Gosque. 


\subsection{Complejo Endorreido de Lebrija-Las Cabezas}

Su localización en una zona eminentemente agrícola, si bien ha permitido la conservación de pequeñas manchas de matorral meditarráneo en el área de las cuencas vertientes, ha motivado una amplia transformación y degradación de la vegatación perilagunar. Son graves los problemas de erosión y colmatación de los vasos lagunares, así como, en algunos casos, su invasión por cultivos, particularmente preocupantes en la laguna de Charroao. Destaca por su perfecto estado de conservación el extenso tarajal que se desarrolla en la periferia de Cigarrera, muestra de la vegetación que antaño debía rodear al resto de las lagunas del complejo.

La presencia de ganado no es preocupante, ya que tan sólo eventualmente acuden rebaños caprinos y ovinos a las inmediaciones de Cigarrera.

El comportamiento hídrico no parece estar gravemente alterado ante la escasez de pozos en la zona y tan sólo en Cigarrera, Galiana y Taraje existen extracciones de poca trascendencia en el ámbito de las cuencas vertientes si bien, como ya ha quedado apuntado, deben realizarse estudios específicos a fin de conocer con exactitud el papel de las aguas subterráneas.

Deben comentarse por su valor histórico y anecdótico los intentos de transformación sufridos por las lagunas de Peña, donde se observan algunos pies de eucaliptos plantados en el vaso lagunar quizás con el propósito de desecarla, y en Taraje, donde aún se conserva una compuerta, restos de un antiguo intento de desecación.

Mayor trascendencia poseen los impactos derivados de las infraestructuras presentes en el área. El complejo es atravesado en la actualidad por un tendido eléctrico de baja tensión, que ha causado la electrocución de diversas aves en las proximidades de la laguna del Pilón.

\subsection{Complejo Endorreico de Utrera}

Las lagunas Zarracatín, Arjona y Alcaparrosa están sometidas a diversos impactos relacionados con la total transformación del área en cultivos. Este hecho conlleva problemas de erosión y colmatación, principalmente impactantes en Zarracatín, debido a la poca profundidad de la misma y la práctica ausencia de vegetación perilagunar.

La existencia de cultivos de regadíos al norte de Alcaparrosa implican que las aguas sobrantes sean vertidas a la laguna, lo que altera sensiblemente sus características naturales y su comportamiento hídrico, al permanecer con agua artificialmente durante gran parte del año.

Tanto los vertidos directos como el lavado de nutrientes en las cuencas de las distintas lagunas provocan problemas de eutrofización. 
Dentro de los impactos infraestructurales potenciales debe anotarse que el espacio es atravesado por un Oleoducto subterráneo, que pasa cercano a las lagunas de Alcaparrosa y Zarracatín.

\subsection{Complejo Endorreico de La Lantejuela}

Los principales impactos en el área derivan también de las actividades agrícolas que han ocasionado erosión en las cuencas, degradación de la vegetación perilagunar, colmatación de los vasos y problemas de eutrofización. Hasta hace pocos años Ballestera recibía vertidos de piedras provenientes de los cultivos cercanos, provocando colmatación en su lado sur-oriental y posiblemente alteración en las características de sus aguas.

La cercanía de la carretera comarcal 430 a la lalguna de Calderón chica, junto a la inexistencia de vegetación perilagunar, parecen afectar negativamente a las poblaciones de aves acuáticas que visitan la laguna.

En años lluviosos muchas especies de aves hacen uso de las lagunas de Calderón Grande y Turquilla, cercanas a las de La Lantejuela, por lo que ambas, aunque en la actualidad no se encuentren protegidas, podrían jugar un papel importante para la avifauna.

\subsection{Laguna de El Gosque}

La laguna del Gosque está sujeta a diversos procesos que vienen modificando sus características naturales, siendo especialmente impactante la alteración del régimen hídrico, mediante la canalización de los arroyos que drenaban a la misma y la existencia de más de una veintena de pozos en sus inmediaciones, con una considerable mengua en su extensión en los últimos decenios.

El uso de abonos y productos fitosanitarios producen una alta eutrofización de sus aguas, mientras que son corrientes los vertidos de piedras en el vaso lagunar, a lo que debe unirse los problemas de erosión, generalizados en toda la cuenca.

Por último, debe recordarse su uso como zona de baño. Aunque esta actividad no se desarrolla hoy en día, apunta la relación existente entre la laguna y el medio social en el que se enclava, por lo que la participación ciudadana en la gestión del espacio adquiere mayor peso específico que en otras zonas.

\section{GESTIÓN Y CONSERVACIÓN}

La importancia de las zonas húmedas interiores para las comunidades de avifauna acuática ha motivado que tradicionalmente éste haya sido el factor determinante a la hora de diseñar su conservación y gestión. 
Este hecho ha demostrado sin embargo diversos inconvenientes. En primer lugar puede subvalorar ambientalmente un humedal, al no tener en cuenta otros factores (geomorfológicos, históricos, económicos, bióticos, etc.), como puede ser el caso, anteriormente comentado, de la vegatación acuática asociada a los mismos (TABLA IV). Además, puede traer consecuencias no previstas sobre la estructura y dinamismo del ecosistema, como se ha puesto de manifiesto para el caso del flamenco en Doñana, y que puede ser extrapolado para ésta y otras especies (limícolas) a otras lagunas salinas (MONTES y BERNUES, 1991)

Las especiales características de estos ecosistemas implican que su gestión deba realizarse de forma integrada, basándose más en conocer su funcionamiento que en la presencia de determinadas especies. Desde esta perspectiva más global, el desarrollo de modelos que realicen el funcionamiento de las zonas húmedas adquiere especial interés. Dos aportaciones pueden destacarse:

A) El carácter temporal de la mayoría de estos sistemas posibilita que su funcionamiento y el uso del espacio por las diversas especies pueda ser analizado utilizando la modelística de los ecosistemas fluctuantes, que permite introducir el papel de la energía externa y considerar como esencial la sucesión (MARGALEF, 1987).

TABLA IV

VALORACIÓN DE LAS LAGUNAS DE LA PROVINCIA SEGÚN LA UTILIZACIÓN DE CRITERIOS AVIFAUNÍSTICOS O BOTÁNICOS

\begin{tabular}{llll}
\hline & $\begin{array}{l}\text { Presencia aves } \\
\text { amenazadas }\end{array}$ & $\begin{array}{l}\text { Presencia aves } \\
\text { protegidas }\end{array}$ & Importancia Botánica \\
\hline $\begin{array}{l}\text { Taraje } \\
\text { Pilón }\end{array}$ & Nacional/Regional & Regional & Importancia Nacional \\
Cigarrera & - & - & Importancia Nacional \\
Peña & Nacional/Regional & Regional & - \\
Galiana & - & - & - \\
Charroao & - & - & Importancia Nacional \\
Zarracatín & - & Regional & Interés Singular \\
Arjona & - & Nacional/Regional & - \\
Alcaparrosa & - & - & - \\
Calderón Chica & - & Regional & Imp.Nacional/Int.Singular \\
Ballestera & - & Regional & Imp.Nacional/Int.Singular \\
Gosque & - & Nacional/Regional & Imp.Nacional/Int.Singular \\
\hline
\end{tabular}

Fuente: AMAT et al. (1985) y CIRUJANO et al. (1992). 
B) El diseño de modelos integradores del flujo de materia y energía (FIGURA III), de modo análogo a los realizados para otras zonas húmedas interiores como en Fuente de Piedra (NIELL y LUCENA, 1986). Esta aproximación permite definir las principales variables del ecosistema y diseñar las prioridades de investigación y uso, con vistas a la gestión integral.

\section{CONCLUSIONES}

Las lagunas endorreicas protegidas de Sevilla son una pequeña muestra deI rosario de humedales que hasta hace poco tiempo se diseminaban por la superficie de la provincia. Unos pocos ejemplos de la enorme riqueza perdida son: Ruíz Sánchez, Calderón y Ojuelos (en Osuna), las lagunas de Arroyuelos y Santo Domingo (en Carmona), la de Zahariche (en el término de Fuentes de Andalucía), la laguna de Sevilla (en Marchena), la laguna de Candilejo (en Cabezas de San Juan), o las lagunas de Val de Ojo, Vocesa, Malagueño y Moro (en Lebrija).

En las últimas décadas, el interés por las zonas húmedas ha provocado una especial atención tanto desde el punto de vista científico como legislativo. Sin embargo su importancia para la avifauna acuática ha constituido en ocasiones el factor determinante para su protección, dejando en un segundo plano su trascendencia como reservorios de flora, su carácter cultural o su valor paisajístico y estético que son por sí mismos factores que determinarían la necesidad de su preservación.

La consideración de espacios protegidos de estas lagunas y la labor realizada por diversos organismos, Agencia de Medio Ambiente de la Junta de Andalucía y distintos Ayuntamientos, permite observar con optimismo su conservación. No obstante aún son diversos los problemas que les afectan, derivados fundamentalmente por su localización en zonas agrícolas. La erosión y degradación de suelos, la alteración de las características de sus aguas y la modificación del régimen hídrico, son los que poseen mayor trascendencia, sin que sea conocida con exactitud la repercusión de estos procesos sobre los distintos componentes del ecosistema ni la respuesta del sistema en su conjunto.

Es imprescindible pues, para la preservación a largo plazo, el desarrollo de estudios específicos con el fin de obtener una visión global del funcionamiento del ecosistema con la mayor aproximación posible. Los datos aportados en el presente trabajo, pese a las insuficiencias señaladas, reflejan una realidad que puede ser útil tanto para mostrar el estado ambiental de los diferentes espacios, como para evaluar su evolución en el tiempo y valorar la eficacia de las medidas de gestión adoptadas. 

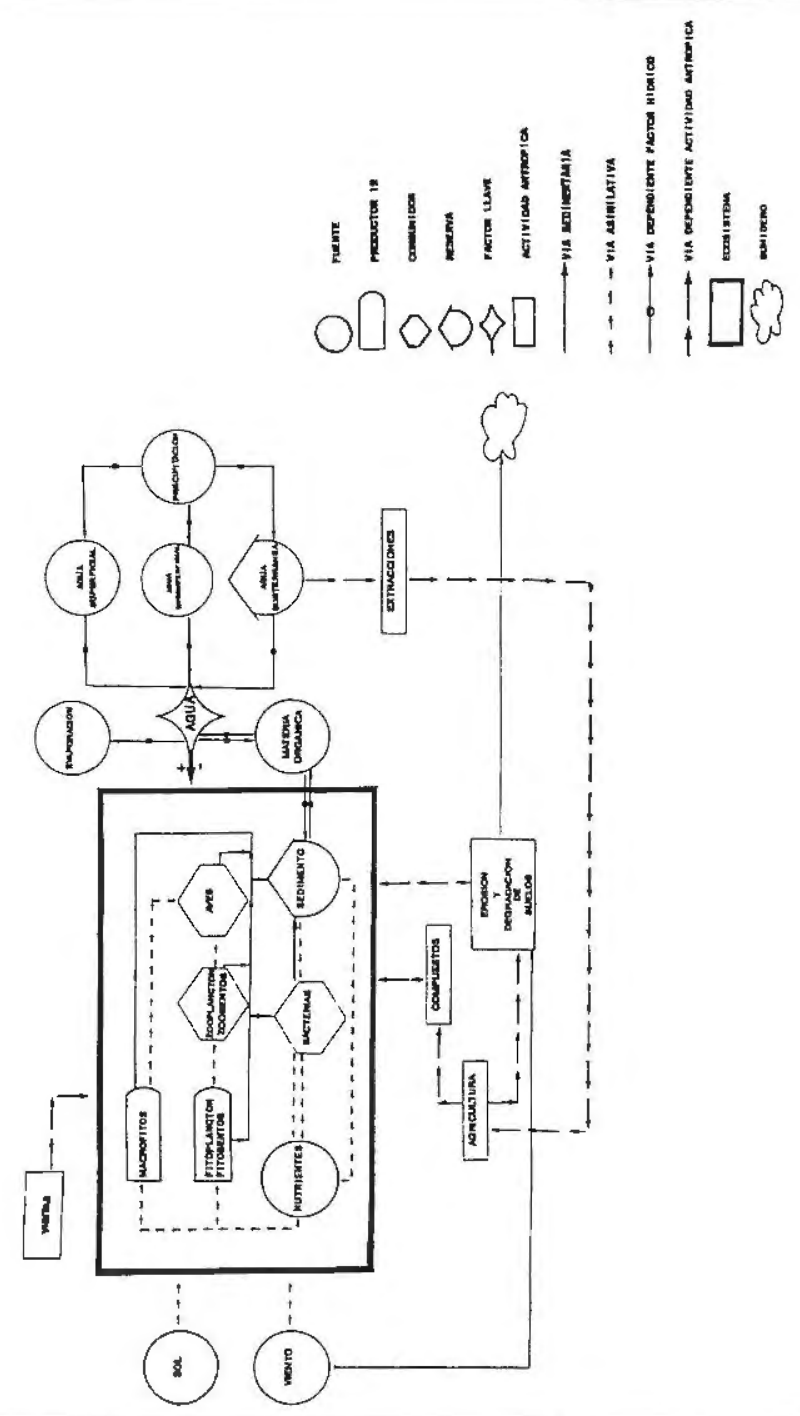

En base al trabajo realizado por NIELLS y LUCENA (1986) para la laguna de Fuente de Piedra, se ha diseñado un modelo de similares características para la laguna sevillana de El Gosque. En él se definen las principales variables del ecosistema, de modo que el conocimiento de las mismas permita hacer estimaciones sobre la respuesta del sistema ante la modificación de alguna de ellas y su utilidad a la hora de diseñar las investigaciones ya ha sido demostrada (LUCENA, 1991). 


\section{Agradecimientos}

Los autores han utilizado parte del trabajo de campo realizado al tiempo que se confeccionaban los Planes de Ordenación de Recursos Naturales (información ambiental) de los espacios lagunares. El artículo es una contribución al Proyecto DGICYT PS 93-0105 dirigido por el Dr. F. Díaz del Olmo.

\section{BIBLIOGRAFÍA}

ALONSO, M.; COMELLES, M. y MARGALEF, R. (1980): Nuevas citas para España de Althenia filiformis Petit. Lagascalia 9:220-223.

A.M.A. (1990): Guía de los Espacios Naturales de Andalucía. Sevilla. 190 pp.

A.M.A. CORDOBA (1991): Evolución de la población ibérica de Malvasía (Oxyura leucocephala). Plan Rector de Uso y Gestión de las Reservas Naturales lagunas de Cádiz. A.M.A. Sevilla. pp: $145-151$.

Aunque el modelo se presenta ampliamente simplificado, incluye los principales componentes que determinan el funcionamiento de la laguna. A nivel general pueden establecerse cuatro subsistemas diferenciados: A) Sistema lagunar; B) Conjunto de actividades antrópicas que repercuten en la laguna; C) Subsistema hídrico y D) Fuentes de Energía.

A) Sistema lagunar: Se definen las principales interacciones entre los distintos comportamientos del sistema (tanto bióticos como ahióticos). La inclusión tan sólo de las aves y no de vertebrados en general obedece a las especiales características salinas y de temporalidad de E1 Gosque que no favorece la presencia de otros vertebrados adaptados al medio acuático (p. ej. fauna piscícola o herpetofauna), que si deberán ser incluidos allí donde su presencia pueda jugar un papel destacado.

B) Actividades antrópicas: Se presta especial atención a las derivadas de las acciones agrícolas, ya que son las que mayor repercusión poseen en el ecosistema. Su papel tanto en la modificación del régimen hídrico natural como en la aportación de materia y energía (erosión, compuestos químicos, abonos, etc.) al ecosistema debe ser valorada y cuantificada a la hora de diseñar su regulación.

C) Subssitema hídrico: Es evidentemente uno de los componentes principales, si no el principal, del ecosistema. El conocimiento del comportamiento hídrico de la laguna es fundamental a la hora de acometer la gestión del espacio. Este comportamiento debe aparecer reflejado en el balance bídrico, el cual debe valorar todos los términos de entrada, salida y almacenamiento. La importancia del mismo alcanza especial relevancia ya que constituye la base de los balances de masa, entre los cuales el de nutrientes es fundamental (CUSTODIO, 1987).

D) Fuentes de Energía: Referido a las fuentes naturales y que incluye la energía proveniente del sol y el viento. El papel de la primera es de sobra conocido. La importancia del viento en el funcionamiento del ecosistema merece especial atención en un triple vertiente: 1) Trascendencia en los procesos erosivos y en el balance de ganancias/pérdidas de materia por el sistema; 2) Papel jugado en el movimiento de la masa de agua y remoción de los sedimentos con la consiguiente liberación de nutrientes; y 3 ) Importancia como agente modelador de las depresiones lagunares (GONZALEZ BERNALDEZ; 1987). 
AMAT, J.A. (1984a): Las poblaciones de aves acuáticas en las lagunas andaluzas: Composición y diversidad durante un ciclo anual. Ardeola № 31: 61-79.

AMAT, J.A. (1984b): Ecología de las lagunas andaluzas. Las zonas húmedas en Andalucía. MOPU. Madrid. pp: 107-117.

AMAT, J.A.; PANIAGUA, C.; HERRERA, C.M.; JORDANO, P.; OBESO, J.R.; SORIGUER, R. (1985): Criterios de valoración de zonas húmedas de importancia nacional y regional en función de las aves acuáticas. ICONA. Monografías $n^{2} 35$. Madrid. 79 pp.

ARAUJO, J. (1978): Censo español de aves acuáticas de enero de 1975. Ardeola 24: 121-205.

ARAUJO, J. y GARCÍA RUA. (1974): El censo español de aves acuáticas de enero de 1973. B.E.C.E. 2 (4): 11-39.

ARENAS GONZÁLEZ, R. y AYALA MORENO, J.M. (1986): Censos de aves acuáticas en las lagunas de Sevilla y Cádiz. Oxyura III (I): 147-148.

BERNIS, F. (1972): El censo español de aves acuáticas en Enero de 1972. Ardeola 17/18: $37-$ 77.

BERNIS, F. y VALVERDE, J.A. (1968): El censo español de aves acuáticas del inviemo de 196768. Ardeola 13: 5-23.

BLASCO, M.; LUCENA, J. y RODRÍGUEZ, J. (1979) Los flamencos de Fuente Piedra. Naturalia hispanica $n^{\circ} 23$. ICONA. Madrid. $55 \mathrm{pp}$.

CASADO DE OTAOLA, S y MONTES DEL OLMO, C. (1995): Guía de los lagos y humedales de España. Reyero Editor. Madrid. 255 pp.

CEBALLOS BENITO, J.J. (1987): Censos de aves acuáticas en las lagunas de la provincia de Sevilla y Cádiz (datos correspondientes al trienio 1983-85). Oxyura IV (1): 205-213.

CIRUJANO, S.; VELAYOS, M.; CASTILLA, F. y GIL, M. (1992): Criterios botánicos para la valoración de las lagunas y humedales españoles. ICONA. Serie Técnica. Madrid. $456 \mathrm{pp}$.

COLÓN DÍAZ, M. y DÍAZ DEL OLMO, F. (1990): Guías Naturalistas de la Provincia de Cádiz: Las Campiñas. Diputación Provincial de Cádiz. 259 pp.

CONTRERAS DE VERA, A., LEÓN VIZCAINO, L.; CUBERO PABLO, M.J. (1989): Distribución de esporos botulínicos en lagunas andaluzas. Oxyura V (1): 119-126.

CUSTODIO, E. (1987): Peculiaridades de la hidrología de los complejos palustres españoles. Bases científicas para la protección de los humedales en España. Real Academia de Ciencias Exactas, Físicas y Naturales. Madrid. pp: 43-63.

DANTÍN, J. (1940): La aridez y el endorreismo en España. El endorreismo bético. Estudios Geográficos, $1 . \mathrm{pp}: 75-117$.

DÍAZ DEL OLMO, F.; RECIO ESPEJO J.M.; JIMÉNEZ MONTES, S.; TORRES GIRÓN Ma L. (1990): Depósitos carbonatados y suelos. Sector El Rubio-Osuna. Seminario Suelos, Paleosuelos y Sedimentos correlativos. Universidad de Sevilla. pp: 85-103.

DÍAZ DEL OLMO, F., MOLINA VÁZQUEZ, F. y SANTO-ROSA CARBALLO, R. (1985): Lagunas y complejos endorreicos de Cádiz (España). Revisión naturalística y medioambiental Oxyura II (1): 85-94.

DÍAZ DEL OLMO, F. y RECIO ESPEJO, J.M. (1991): Lagunas y áreas lacustres continentales de Andalucía Occidental. (Geomorfología, Suelos y Evolución Cuaternaria). Cuadernos de Investigación Geográfica. Tomo XVII: 25-36.

ENA, V. y PURROY, F.J. (1982): Censos invernales de aves acuáticas en España (febrero 1978, 1979 y 1980) ICONA. Madrid. 63 pp.

ENA, V. y PURROY, F.J. (1983): Resultado del censo de aves acuáticas (Enero 1983). Ardeola 30: $100-105$.

FAJARDO, A. y MAZUELOS, M. (1983): El área endorreica de La Lantejuela. Réquiem por una zona húmeda de importancia internacional. Quercus $N^{o}$ 9: 34-36. 
FAJARDO, A. y MAZUELOS, M. (1993): Oportunidades de restauración de humedales endorreicos en Andalucía: el complejo endorreico de La Lantejuela. Seminario sobre bases científicas para la regeneración de humedales. Organizado por la A.,M.A. Junta de Andalucía.

FERNÁNDEZ-PALACIOS, J.M. y RAYA, C. (1991): Biología de la focha cornuda en Cádiz y otros humedales del Bajo Guadalquivir. Plan Rector de Uso y Gestión de las Reservas $\mathrm{Na-}$ turales Lagunas de Cádiz. A.M.A.: 97-117.

FUREST, A. y TOJA, J. (1984): Reconocimiento limnológico de las lagunas andaluzas. Las Zonas Húmedas en Andalucía. MOPU. Madrid. pp: 177-188.

FUREST, A. y TOJA, J. (1987): Tipificación de las lagunas andaluzas según sus comunidades de crustáceos. Oxyura IV (1): 89-100.

GARCÍA MURILLO, P. (1991): Aspectos de la vegetación de las lagunas gaditanas. Plan Rector de Uso y Gestión de las Reservas Naturales Lagunas de Cádiz A.M.A. pp: 47-71.

GARCÍA, L.; AMAT, J.A. y SÁNCHEZ A. (1980): Resultado de los censos de aves acuáticas en Andalucía Occidental durante el invierno 1978-79. Doñana Act. Vert. 7 (1): 19-27.

GONZÁLEZ BERNÁLDEZ, F. (1987): Las zonas húmeđas. El marco conceptual. Bases científicas para la protección de los humedales en España. Real Academia de Ciencias Exactas, Físicas y Naturales. Madrid. pp: 9-30.

HAFNER, H. y WALMSLEY, J. (1974): El censo de aves acuáticas en las marismas del Guadalquivir invierno 1973-74 con datos de otras localidades de Andalucía. Ardeola 20: 161-178.

HERNÁNDEZ BERMEJO, J.E. y CLEMENTE MUÑOZ, M. (1994): Táxones vegetales andaluces (A nivel de Especie y Subespecie) considerados en la categoría de máximo riesgo de extinción. Protección de la Flora de Andalucía. AMA. Sevilla. pp: 67-100.

I.G.M.E. (1988): Hoja № 1.020 (El Coronil) del mapa geológico de España 1:50.000. Madrid. $32 \mathrm{pp}+$ mapa.

LUCENA, J. (1991): Estudio integral de la laguna de Fuentes de Piedra (Málaga España) como vía de conservación de la misma y sus repercusiones sobre el flamenco (Phoenicopterus ruber roseus Pall.) Reunión técnica del flamenco rosa (Phoenicopterus ruber roseus) en el Mediterráneo Occidental y Africa Noroccidental. A.M.A. Sevilla. pp: 185-196.

MACHADO, A. (1854): Catálogo de las aves observadas en algunas de las provincias de Andalucia. Sevilla. universidad de Sevilla.

MADOZ, P. (1845): Diccionario geográfico histórico estadístico de españa y sus posesiones de Ultramar. Edición Faccimil. Ambito Ediciones. Sevilla. 398 pp.

MOLINA VÁZQUEZ, F. (1991): Gestión de complejos endorreicos: su aplicación en las lagunas de la provincia de Cádiz. P.R.U.G. de las Reservas Naturales de las Lagunas de Cádiz. A.M.A. Sevilla. pp: 173-184.

MONTES, C.; AMAT, J.A. y RAMÍREZ DÍAZ, L. (1982a): Ecosistemas acuáticos del Bajo Guadalquivir (SW España) I. Características generales, físico-químicas y biológicas de las aguas. Studia Oecologica. III: 129-158.

MONTES, C.; AMAT, J.A. y RAMÍREZ DÍAZ, L. (1982b): Ecosistemas acuáticos del Bajo Guadalquivir (SW España)II. Variación estacional de los componenetes físico-químicos y biológicos de las aguas. Studia Oecologica. III: 159-180.

MONTES, C. y MARTINO, P. (1987): Las lagunas salinas españolas. Bases científicas para la protección de los humedales en España. Real Academia de Ciencias Exactas, Físicas y Naturales. Madrid. pp: 95-145. 
MONTES, C. y BERNUES, M. (1991): Incidencia del flamenco rosa (Phoenicopterus ruber roseus) en el funcionamiento de los ecosistemas acuáticos de la marisma del Parque Nacional de Doñana. Reunión técnica ciel flamenco rosa (Phoenicopterus ruber roseus) en el Mediterráneo Occidental y Africa Noroccidental. A.M.A. Sevilla. pp: 103-110.

NIELL, F.X. y LUCENA, J.C. (1986): Comentarios sobre un sistema único: la laguna de Fuente de Piedra, ideas de como planificar su uso y gestión. OXYURA III (I): 35-42.

ORTIZ BOTELLA, F.J. y MOLINA VAZQUEZ, J. (1995): Características naturales y problemas de conservación de la laguna de El Gosque Quercus 117: 40-41.

PARDO, L. (1948): Catálogo de los lagos de España. Ins. Forestal Inv. y Exp. Madrid. 523 pp.

PEREIRA SIESO, P. (1991): Resultados del seguimiento de malvasías procedentes del programa de cría en cautividad durante el año 1990. Plan Rector de Uso y Gestión de las Reservas Naturales Lagunas de Cádiz. A.M.A.: 135-143.

RAMíREZ, J.M., RENDON, M. y VARGAS, J.M. (1992): Reproducción e invernada de la malvasía (Oxyura leucocephala) en las lagunas endorreicas de la provincia de Málaga. Oxyura $V I(1): 55-56$.

RECIO ESPEJO, J.M. (1989): Endorreísmo y lagunas andaluzas. El Cuaternario en Andalucía Occidental. AEQUA Monografías № 1. Sevilla. pp: 99-104.

REYES PROSPER, E. (1915): Las estepas de España y su vegetación. Ed. Casa Real, Madrid. $305 \mathrm{pp}$.

SÁNCHEZ MORENO, A. (1979): Resultados de los censos de aves acuáticas invernantes en el Sur-oeste de España. Inviernos 1975-76 y 1976-77. Doñana Acta Vertebrata VI (1): 67-76.

SEO (1984): Censos invernales de aves acuáticas (1984). La Garcilla 64: 21-22.

SEO (1985): Censo de anátidas y fochas. Resultados de enero 1985 La Garcilla 65: 12-16.

SEO (1987a): Resumen del censo nacional de aves acuáticas invernantes (Enero 1986) La Garcilla 68: $10-14$.

SEO (1987b): Resumen del censo nacional de aves acuáticas invernantes (Enero 1987) La Garcilla 70: $10-13$.

SEO (1990): Resumen del censo nacional de aves acuáticas invernantes. Enero 1988. La Garcilla 77: 6-7 y 28.

SILVESTRE, S. y CABEZUDO, B. (1980): Notas taxonómicas y corológicas sobre la vegetación de Andalucía Occidental. Lagascalia 4 (2): 247.

TALAVERA, S.; AMAT, J.A.; FUREST, A. (1984): Notas taxonómicas y corológicas sobre la Flora de Andalucía occidental: Althenia filiformis Petit. Lagascalia 12: 252-253.

TORRES ESQUIVIAS, J.A.; MORENO ARROYO, B. y ALCALA-ZAMORA, A. (1994): La población española de malvasía cabeciblanca (Oxyura leucocephala) a la luz de los últimos censos nacionales. Oxyura VII (I): 89-108. 